\title{
The inhibition of c-MYC transcription factor modulates the expression of glycolytic and glutaminolytic enzymes in FaDu hypopharyngeal carcinoma cells
}

\author{
Robert Kleszcz ${ }^{A-D}$, Jarosław Paluszczak ${ }^{A-C, E, F}$, Violetta Krajka-Kuźniak ${ }^{B}$, Wanda Baer-Dubowska ${ }^{C, E, F}$ \\ Department of Pharmaceutical Biochemistry, Poznan University of Medical Sciences, Poland \\ A - research concept and design; $\mathrm{B}$ - collection and/or assembly of data; $\mathrm{C}$ - data analysis and interpretation; \\ $D$ - writing the article; $E$ - critical revision of the article; $F$ - final approval of the article
}

Address for correspondence

Wanda Baer-Dubowska

E-mail: baerw@ump.edu.pl

Funding sources

This study was supported by a grant from

Poznan University of Medical Sciences,

№. 502-14-03302403-41154.

Conflict of interest

None declared

\section{Acknowledgements}

The authors would like to thank

prof. dr hab. Marek Murias for obtaining

the DMU-212 compound used in this research.

Received on July 6, 2016

Reviewed on 0ctober 23, 2016

Accepted on February 14, 2017

\section{Abstract}

Background. Cancer cells are dependent on aerobic glycolysis for energy production and increased glutamine consumption. HIF-1a and c-MYC transcription factors regulate the expression of glycolytic and glutaminolytic genes. Their activity may be repressed by SIRT6. Head and neck carcinomas show frequent activation of C-MYC function and SIRT6 down-regulation, which contributes to a strong dependence on glucose and glutamine availability.

Objectives. The aim of this study was to compare the influence of HIF-1a and c-MYC inhibitors (KG-548 and 10058-F4, respectively) and potential SIRT6 inducers - resveratrol and its synthetic derivative DMU-212 with the effect of glycolysis and glutaminolysis inhibitors (2-deoxyglucose and aminooxyacetic acid, respectively) on the metabolism and expression of metabolic enzymes in FaDu hypopharyngeal carcinoma cells.

Material and methods. Cell viability was assessed by means of an MTT assay. Quantitative PCR was performed to evaluate the expression of SIRT6, HIF-1a, C-MYC, GLUT1, SLC1A5, HK2, PFKM, PKM2, LDHA, GLS, and $G D H$. The release of glycolysis and glutaminolysis end-products into the culture medium - lactate and ammonia, respectively - was assessed using standard colorimetric assays.

Results. Lactate production was significantly inhibited by 10058-F4, KG-548, and 2-deoxyglucose. Moreover, 10058-F4 strongly reduced the amount of ammonia release. The effects of 10058-F4 activity can be attributed to a reduction in the expression of PKM2 and LDHA. On the other hand, the induction of SIRT6 expression by resveratrol and DMU-212 was not associated with significant modulation of the expression of metabolic enzymes.

Conclusions. Overall, the results of this study indicate that the inhibition of c-MYC may be considered to be a promising strategy of the modulation of cancer-related metabolic changes in head and neck carcinomas.

Key words: c-MYC, energy metabolism, the Warburg effect, 10058-F4, FaDu cells

DOI

10.17219/acem/68979

Copyright

Copyright by Author(s)

This is an article distributed under the terms of the

Creative Commons Attribution Non-Commercial License

(http://creativecommons.org/licenses/by-nc-nd/4.0/) 


\section{Introduction}

Cancer cells, in contrast to normal cells, generate energy by increasing aerobic glycolysis, a phenomenon termed "the Warburg effect". ${ }^{1}$ Altered energy metabolism supporting continuous cell growth and proliferation was pointed out as a new hallmark of cancer cells. ${ }^{2}$ Several hypotheses have been proposed to explain the maintenance of this seemingly wasteful catabolic state. Recent investigations into the mechanisms that underlie the Warburg effect indicate that beside the direct involvement of overexpressed uncoupling proteins (UCP) in the Warburg effect, the alterations in the glycolytic pathway itself may be equally or even more important. In this regard, it has been suggested that pyruvate kinase M2 (PKM2) or hexokinase 2 (HK2) might be the key mediators of aerobic glycolysis and promote tumor growth, at least in certain types of tumors. ${ }^{3-5}$ Moreover, PKM2 was shown to function as a co-activator of hypoxia inducible factor 1 (HIF-1). ${ }^{6}$

The HIF-1 factor may be considered a driver molecule of glycolytic control. It is composed of an HIF-1 $\alpha$ subunit, which is susceptible to changes in oxygen concentration (stabilized in hypoxic conditions), and HIF-1 $\beta$ being constitutively expressed. ${ }^{7}$ Typically, HIF- $1 \alpha$ is expressed in hypoxia, whereas under oxygen stimuli, prolyl hydroxylase 2 (PHD2) promotes HIF-1 $\alpha$ association with von Hippel-Lindau tumor suppressor (VHL), which finally leads to the ubiquitination and proteasomal degradation of HIF- $1 \alpha .{ }^{8,9}$ However, oncogenic $\mathrm{H}$-Ras and phosphatidylinositol 3-kinase signaling can maintain an elevated HIF- $1 \alpha$ level even in aerobic conditions. ${ }^{9}$ Moreover, inactivating mutations of genes encoding succinate dehydrogenase and fumarase lead to an accumulation of succinate and fumarate, which results in the stabilization of HIF- $1 \alpha$ in normoxia via the inhibition of prolyl hydroxylase., ${ }^{7,9}$

There are also suggestions that aerobic glycolysis may represent a shift to the oxidative metabolism of non-glucose carbon sources, particularly glutamine - an amino acid that is ultimately converted to $\alpha$-ketoglutarate in the mitochondria to enter the citric acid cycle (CAC). ${ }^{4}$ Indeed, glutamine seems to be a nutrient as important as glucose for some types of malignant cells. For instance, the proliferation and survival of head and neck squamous cell carcinoma cells (HNSCC) strongly depend on both glucose and glutamine availability, where cell proliferation can be maximized only in the presence of glutamine. ${ }^{10}$ The $\mathrm{c}-\mathrm{MYC}$ transcription factor is suggested to be important in the regulation of both glutamine and glucose metabolism. ${ }^{11}$ Cells overexpressing $c-M Y C$ are glutamine-addicted, which is related to an increased glutaminase 1 (GLS1) level. ${ }^{12} \mathrm{c}-\mathrm{MYC}$ is responsible for the direct promotion of the expression of glutamine transporters enhancing glutamine's cellular entry ${ }^{11,12}$ and is capable of supporting HIF-1 $\alpha$ functions by up-regulating, e.g., $H K 2$, phosphofructokinase (PFK1), PKM2, and lactate dehydrogenase $(L D H)$, contributing to the enhancement of glycolysis intensity as well. ${ }^{8}$
The transcriptional activity of HIF- $1 \alpha$ and c-MYC may be regulated by an interaction with other proteins including epigenetic modulatory proteins. Sirtuins have recently emerged as important regulators of energy metabolism in mammalian cells. ${ }^{13}$ Sirtuins (SIRTs) belong to Class III of the histone deacetylase (HDACs) family and are the only HDACs that require $\mathrm{NAD}^{+}$for their proper enzymatic activity ${ }^{14}$ Histone acetylation is known to promote the local transcriptional activity of chromatin, whereas histone deacetylases, by removing acetyl groups, lead to the formation of tightly packed and transcriptionally inactive heterochromatin. SIRT6 is thought to be especially important in metabolic orchestration, and its depletion may be connected with the development of a metabolic environment conducive to cancer. Both HIF-1 $\alpha$ and SIRT6 influence the expression of several crucial glycolytic genes, e.g., $P F K 1$, aldolase $(A L D)$, pyruvate dehydrogenase kinase 1 (PDK1), LDH and, additionally, glucose transporter GLUT-1. ${ }^{15}$ Lack of SIRT6 leads to the up-regulation of glycolytic genes via HIF-1 $\alpha$ derepression. Similarly, a decreased expression of SIRT6 relieves the repression of c-MYC transcriptional activity. Altogether, SIRT6 depletion may result in the enhancement of HIF- $1 \alpha$ and c-MYC target gene expression, which contributes to a characteristic glucose (HIF-1 $\alpha$ and c-MYC) ${ }^{16}$ and glutamine (c-MYC) addiction. ${ }^{17}$ Importantly, the level of the expression of SIRT6 is decreased in head and neck squamous cell carcinomas. ${ }^{18}$ The activity of SIRT1 may be induced by resveratrol and possibly other stilbene derivatives, and SIRT1 may indirectly induce the expression of SIRT6. ${ }^{19}$

Head and neck squamous cell carcinomas (HNSCCs) are the fifth most common type of cancer ${ }^{20}$ characterized by a poor survival rate, particularly for patients with aggressive tumors, and regional and distant metastases. ${ }^{21} \mathrm{~A}$ better understanding of tumor metabolism in HNSCCs may provide new therapeutic strategies for the treatment of this type of cancer. The data on the regulation of energetic metabolism in HNSCCs, although limited, ${ }^{22}$ suggests that head and neck cancer cell proliferation depends on glucose and glutamine metabolism. The aim of this study was to evaluate the influence of small molecules - inhibitors of specific regulators of both c-MYC inhibitor (10058-F4) and HIF-1 inhibitor (KG-548) pathways - on the proliferation and metabolism of the hypopharyngeal carcinoma FaDu cells. Direct inhibitors of glycolysis (2-deoxyglucose) and transamination (aminooxyacetic acid) were used for comparison. Additionally, we tested whether resveratrol and its synthetic derivative DMU-212 are capable of inducing SIRT6 and, therefore, affecting energy metabolism in FaDu cells.

\section{Material and methods}

\section{Cell culture and viability assay}

The hypopharyngeal carcinoma FaDu cell line was purchased from ATCC (Manassas, Virginia, USA). The cells were grown in Dulbecco's Modified Eagle's Medium 
(DMEM), containing 10\% fetal bovine serum (FBS) (Biowest SAS, Nuaillé, France) and antibiotics - penicillin and streptomycin $1 \%$ (Sigma-Aldrich, St. Louis, USA) - at $37^{\circ} \mathrm{C}$ in a $95 \%$ humidified and $5 \% \mathrm{CO}_{2}$ atmosphere.

The effects of KG-548, 10058-F4, 2-deoxyglucose, resveratrol (Sigma-Aldrich, St. Louis, USA), and DMU-212 (obtained from the Department of Toxicology, Poznań University of Medical Sciences) and aminooxyacetic acid (MP Biomedicals, Illkirch, France) on FaDu cell viability were assessed by the MTT assay according to standard protocols. Briefly, $10^{4}$ cells were seeded per well in a 96 well plate. After $24 \mathrm{~h}$ of pre-incubation in DMEM supplemented with $5 \%$ FBS and antibiotics, the tested compounds were added to the culture medium in various concentrations and the cells were incubated for $24 \mathrm{~h}$. Afterwards, the cells were washed twice with PBS buffer, and a fresh medium containing MTT salt $(0.5 \mathrm{mg} / \mathrm{mL})$ was added. After $4 \mathrm{~h}$ of incubation, formazan crystals were dissolved in acidic isopropanol and absorbance was measured at 570 and $690 \mathrm{~nm}$. All the experiments were repeated 3 times with 3 measurements per assay. In all the subsequent experiments, non-toxic concentrations (viability level $>70 \%$ ) of the compounds were used. The chemical structure of the studied compounds is presented in Fig. 1.<smiles>Oc1ccc(/C=C/c2cc(O)cc(O)c2)cc1</smiles>

resveratrol<smiles>FC(F)(F)c1cc(-c2nn[nH]n2)cc(C(F)(F)F)c1</smiles>

KG-548<smiles>OC[C@H]1OC(O)C[C@@H](O)[C@@H]1O</smiles>

2-deoxyglucose<smiles>COc1ccc(/C=C/c2cc(OC)c(OC)c(OC)c2)cc1</smiles>

DMU-212

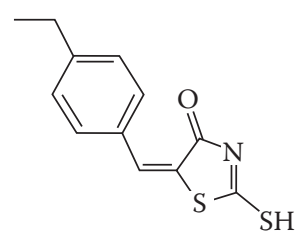

$10058-F 4$<smiles>NOCC(=O)O</smiles>

aminooxyacetic acid

Fig. 1. The chemical structure of the tested compounds

\section{Isolation of total RNA and CDNA synthesis}

$1 \times 10^{6}$ cells were seeded in $100-\mathrm{mm}$ culture dishes, and after $24 \mathrm{~h}$ of pre-incubation in DMEM containing 5\% FBS, the cells were treated with $10 \mu \mathrm{M}$ of resveratrol, $2 \mu \mathrm{M}$ of DMU-212, $50 \mu \mathrm{M}$ of KG-548 and 10058-F4, 1 or $2 \mathrm{mM}$ of 2-deoxyglucose (2-DG), and 0.1 or $0.2 \mathrm{mM}$ of aminooxyacetic acid (AOAA) or vehicle control. After a 24-h incubation, total RNA was isolated using a Universal RNA Purification Kit (EURx, Gdańsk, Poland) and subsequently subjected to reverse transcription using a RevertAid First Strand cDNA Synthesis Kit (Thermo Fisher Scientific Inc., Waltham, USA), according to the manufacturer's recommendations.

\section{Quantitative real-time polymerase chain reaction}

Quantitative real-time polymerase chain reaction (PCR) was performed using the Maxima SYBR Green qPCR Master Mix (2X) (Thermo Fisher Scientific, Waltham, USA)

Table 1. The sequence of starters used in real-time PCR reactions

\begin{tabular}{|c|c|c|c|}
\hline \multicolumn{2}{|c|}{ Primer } & Sequence & Product size \\
\hline \multirow{2}{*}{$P B G D$} & forward & 5'CCGCATCTGGAGTTCAGGAGTATTC & \multirow{2}{*}{$101 \mathrm{bp}$} \\
\hline & reverse & 5'CCAGCTGTTGCCAGGATGATG & \\
\hline \multirow{2}{*}{ TBP } & forward & 5'GGCACCACTCCACTGTATC & \multirow{2}{*}{$183 \mathrm{bp}$} \\
\hline & reverse & 5'GGGATTATATTCGGCGTTTCG & \\
\hline \multirow{2}{*}{ SIRT6 } & forward & 5'ACTGGCGAGGCTGGTCTG & \multirow{2}{*}{$157 \mathrm{bp}$} \\
\hline & reverse & 5'GCTCTCAAAGGTGGTGTCG & \\
\hline \multirow{2}{*}{ HIF-1a } & forward & 5'CAGTAACCAACCTCAGTGTG & \multirow{2}{*}{$199 \mathrm{bp}$} \\
\hline & reverse & 5'AAGTTCTTCTGGCTCATATCC & \\
\hline \multirow{2}{*}{$c-M Y C$} & forward & 5'TTACAACACCCGAGCAAG & \multirow{2}{*}{$133 \mathrm{bp}$} \\
\hline & reverse & 5'AATCCAGCGTCTAAGCAG & \\
\hline \multirow{2}{*}{ GLUT1 } & forward & 5'GCCAAGAGTGTGCTAAAG & \multirow{2}{*}{$107 \mathrm{bp}$} \\
\hline & reverse & 5'ATGGTGACCTTCTTCTCC & \\
\hline \multirow{2}{*}{ SLC1A5 } & forward & 5'CTGCCTTTGGGACCTCTTC & \multirow{2}{*}{$93 \mathrm{bp}$} \\
\hline & reverse & 5'AACGGCTGATGTGCTTGG & \\
\hline \multirow{2}{*}{ HK2 } & forward & 5'GTCCGTAACATTCTCATCG & \multirow{2}{*}{$125 \mathrm{bp}$} \\
\hline & reverse & 5'AGGCAGTCACTCTCAATC & \\
\hline \multirow{2}{*}{ PFKM } & forward & 5'GCCCGTGTCTTCTTTGTC & \multirow{2}{*}{$161 \mathrm{bp}$} \\
\hline & reverse & 5'AGTCGTCCTTCTCGTTCC & \\
\hline \multirow{2}{*}{ PKM2 } & forward & 5'AGAGAAGGGAAAGAACATCAAG & \multirow{2}{*}{$175 \mathrm{bp}$} \\
\hline & reverse & 5'GCACCGTCCAATCATCATC & \\
\hline \multirow{2}{*}{ LDHA } & forward & 5'TCAGCCCGATTCCGTTACC & \multirow{2}{*}{119 bp } \\
\hline & reverse & 5'ACATTCATTCCACTCCATACAGG & \\
\hline \multirow{2}{*}{ GLS } & forward & 5'ATGATGTGCTGGTCTCCTC & \multirow{2}{*}{$177 \mathrm{bp}$} \\
\hline & reverse & 5'ATTTATCACTGACTTTACCCTTTG & \\
\hline \multirow{2}{*}{$\mathrm{GDH}$} & forward & 5'GGATTCTAACTACCACTTGCTC & \multirow{2}{*}{$171 \mathrm{bp}$} \\
\hline & reverse & 5'GAACGCTCCATTGTGTATGC & \\
\hline
\end{tabular}


on a Chromo4 thermal cycler (BioRad Laboratories, Hercules, USA). The primer sequences which are listed in Table 1 were generated using Beacon Designer software and synthesized at the Institute of Biochemistry and Biophysics, Warszawa, Poland. All the reactions were run in triplicate. The protocol started with a 10 min enzyme activation at $95^{\circ} \mathrm{C}$, followed by 40 cycles of $95^{\circ} \mathrm{C}$ for $15 \mathrm{~s}, 56^{\circ} \mathrm{C}$ for $20 \mathrm{~s}$, $72^{\circ} \mathrm{C}$ for $40 \mathrm{~s}$, and the final elongation at $72^{\circ} \mathrm{C}$ for $5 \mathrm{~min}$. The melting curve analysis was used to confirm the generation of a single amplification product. Experiments were normalized for the mean expression of the TATAbox binding protein $(T B P)$ and porphobilinogen deaminase $(P B G D)$. The Pfaffl relative method was used for foldchange quantification.

\section{Lactate and ammonia concentration analysis}

Lactate concentration in the culture medium was measured using an L-Lactate Assay Kit, and the ammonia concentration was tested using an Ammonia Assay Kit (ScienCell Research Laboratories, Carlsbad, USA) according to the manufacturer's instructions. Immediately after acquisition, the media samples were divided into 2 portions and 1 was passed through Amicon Ultra 10K filters (Millipore, Cork, Ireland) in order to remove any LDH activity which could interfere with lactate assessment.

\section{Statistical analysis}

Statistical analysis was performed by one-way ANOVA using STATISTICA software (v. 10). The statistical significance between the experimental groups and their respective controls was assessed by Tukey's post hoc test, with $\mathrm{p}<0.05$ considered significant.

\section{Results}

\section{Cell viability analysis}

Cell viability was assessed by the MTT assay (Fig. 2). 2-deoxyglucose reduced cell viability in a dose-dependent manner (Fig. 2C), in contrast to AOAA, which exerted weaker effects (Fig. 2D). KG-548 did not significantly affect
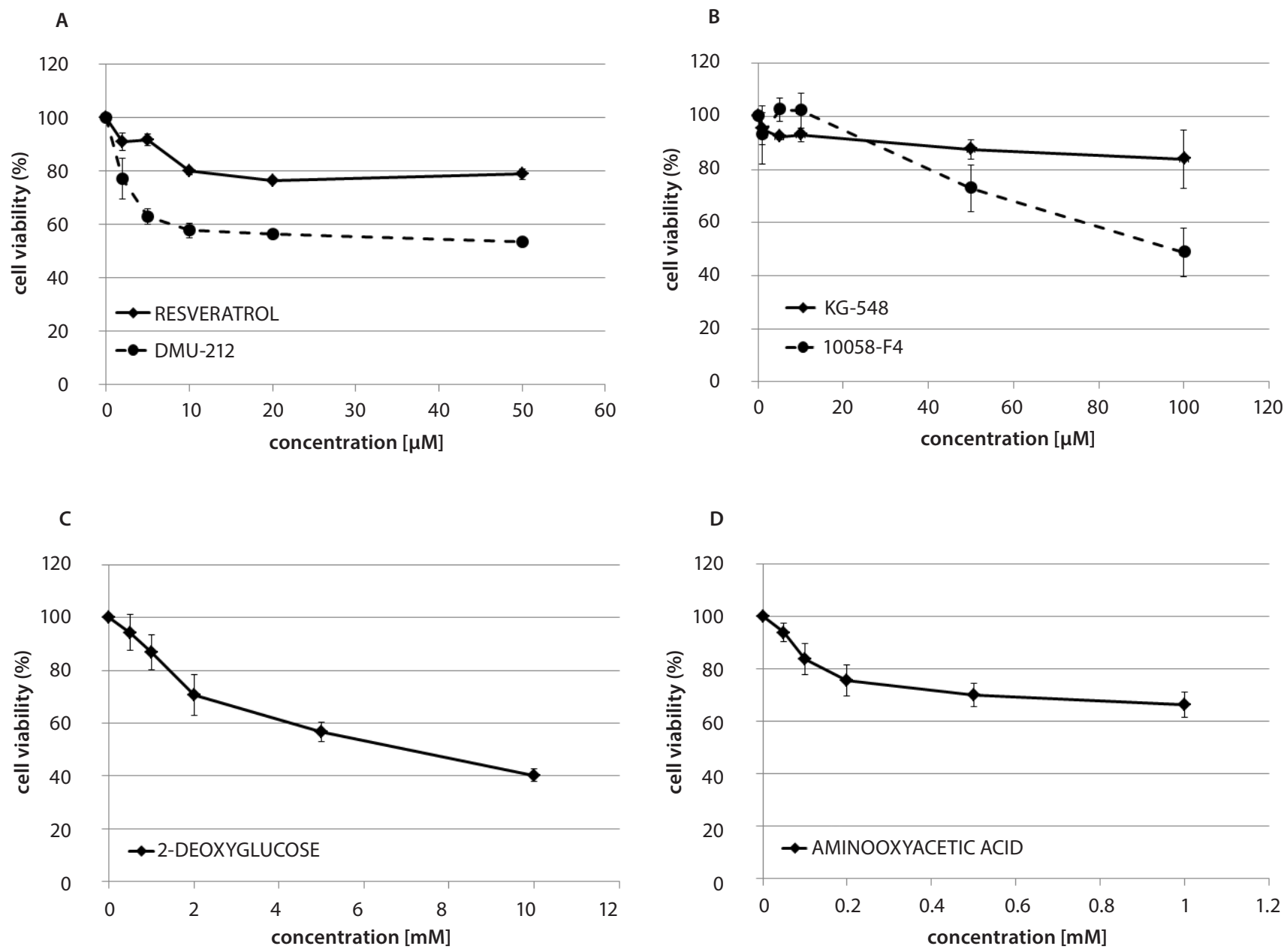

Fig. 2. The effect of the tested compounds on the viability of FaDu cells 

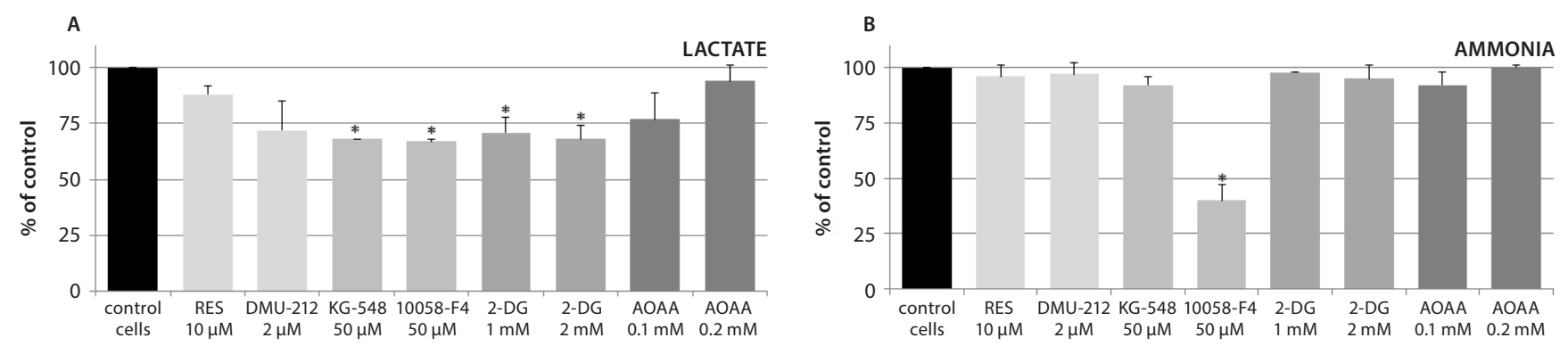

Fig. 3. The effect of resveratrol, DMU-212, and small molecule inhibitors KG-548, 10058-F4, 2-deoxyglucose (2-DG), and aminooxyacetic acid (AOAA) on lactate (A) and ammonia (B) release into the culture medium

Mean values +/- SEM from 2 independent experiments are shown. The level of vehicle-treated cells was considered to be 100\%. An asterisk above a bar denotes statistically significant differences from the control group, $\mathrm{p}<0.05$.

cell viability up to $100 \mu \mathrm{M}$, while 10058-F4 decreased cell viability only at high concentrations (Fig. 2B). DMU-212 showed stronger anti-proliferative effects than its parent compound, resveratrol (Fig. 2A). In all the subsequent experiments, non-toxic concentrations of the tested compounds (viability above $70 \%$ ) were used.

\section{Lactate and ammonia concentration analysis}

The release of glycolysis and glutaminolysis end-products - lactate and ammonia, respectively - into the culture medium was assessed in order to evaluate the effect of the tested modulators on these pathways. Lactate concentration was significantly reduced after treatment with KG-548 (HIF-1 inhibitor), 10058-F4 (c-MYC inhibitor), and 2-deoxyglucose (glycolysis inhibitor). Resveratrol, DMU-212, and AOAA showed a tendency to reduce lactate production; however, the changes were not statistically significant (Fig. 3A). Ammonia production remained unaltered after the FaDu cells were treated with the compounds, with the exception of 10058-F4, which diminished the ammonia level in media samples by almost 60\% (Fig. 3B).

\section{Gene expression analysis}

Figure 4 presents the results of the quantitative PCR which was performed to evaluate the expression of genes associated with the metabolism of glucose (glucose transporter [GLUT1], hexokinase 2 [HK2], phosphofructokinase $\mathrm{M}[P F K M]$, pyruvate kinase M2 [PKM2], and lactate dehydrogenase $\mathrm{A}[L D H A]$ ) and glutamine (glutamine transporter [SLC1A5], glutaminase [GLS], and glutamate dehydrogenase $[G D H])$ and genes which encode regulatory proteins: sirtuin 6 (SIRT6), hypoxia inducible factor $1 \alpha(H I F-1 \alpha)$, and transcription factor $c-M Y C$. Although resveratrol and DMU-212 induced the expression of SIRT6 (Fig. 4A), they did not significantly change the expression of genes related to the metabolism of glucose and glutamine, with the exception of $H K 2$ up-regulation after exposure to DMU-212 (Fig. 4F). KG-548 also did not affect the expression of glucose and glutamine metabolism genes except for GLUT1 (Fig. 4D); however, it did lead to a significant induction of $c-M Y C$ expression (Fig. 4C). The strongest modulation of the expression of the studied genes was exerted by the MYC inhibitor, 10058-F4. This compound decreased the expression of genes engaged in the last stage of glycolysis - PKM2 (Fig. 4H) and LDHA (Fig. 4I) - by approx. $60 \%$. Interestingly, this compound induced the expression of glutamine transporter (Fig. 4E), but had a slight inhibitory effect on the expression of glutaminase (Fig. 4J). Although 2-deoxyglucose reduces lactate generation mainly through the direct inhibition of glycolysis, the treatment of FaDu cells with this compound also led to a decrease in the expression of GLUT1 (Fig. 4D) and HK2 (Fig. 4F).

\section{Discussion}

Head and neck squamous cell carcinomas (HNSCCs) are characterized in most cases by unfavorable outcome rates. Thus, a better understanding of the molecular pathways involved in the malignant transformation of HNSCCs is essential for the development of novel therapies. A shift in glucose degradation from glucose oxidation to aerobic glycolysis and the induction of glutaminolysis are considered to be hallmarks of cancer cell metabolism. However, the data on the regulation of these pathways in HNSCCs are limited and somehow controversial. The aim of this study was to attempt to assess the role of these processes along with SIRT6 activation in FaDu cells derived from hypopharyngeal SCC using small molecule inhibitors of specific target elements of these pathways.

The results pointed to the c-MYC inhibitor, 10058-F4, as the most effective modulator of both pathways. This was the only compound among the tested modulators which decreased both lactate and ammonia production, the endproducts of glycolysis and glutaminolysis, respectively. Moreover, the c-MYC inhibitor decreased the expression of the $L D H A$ gene encoding a major molecular mediator of the Warburg effect as well as the PKM2 gene and slightly decreased glutaminase expression. Interestingly, 

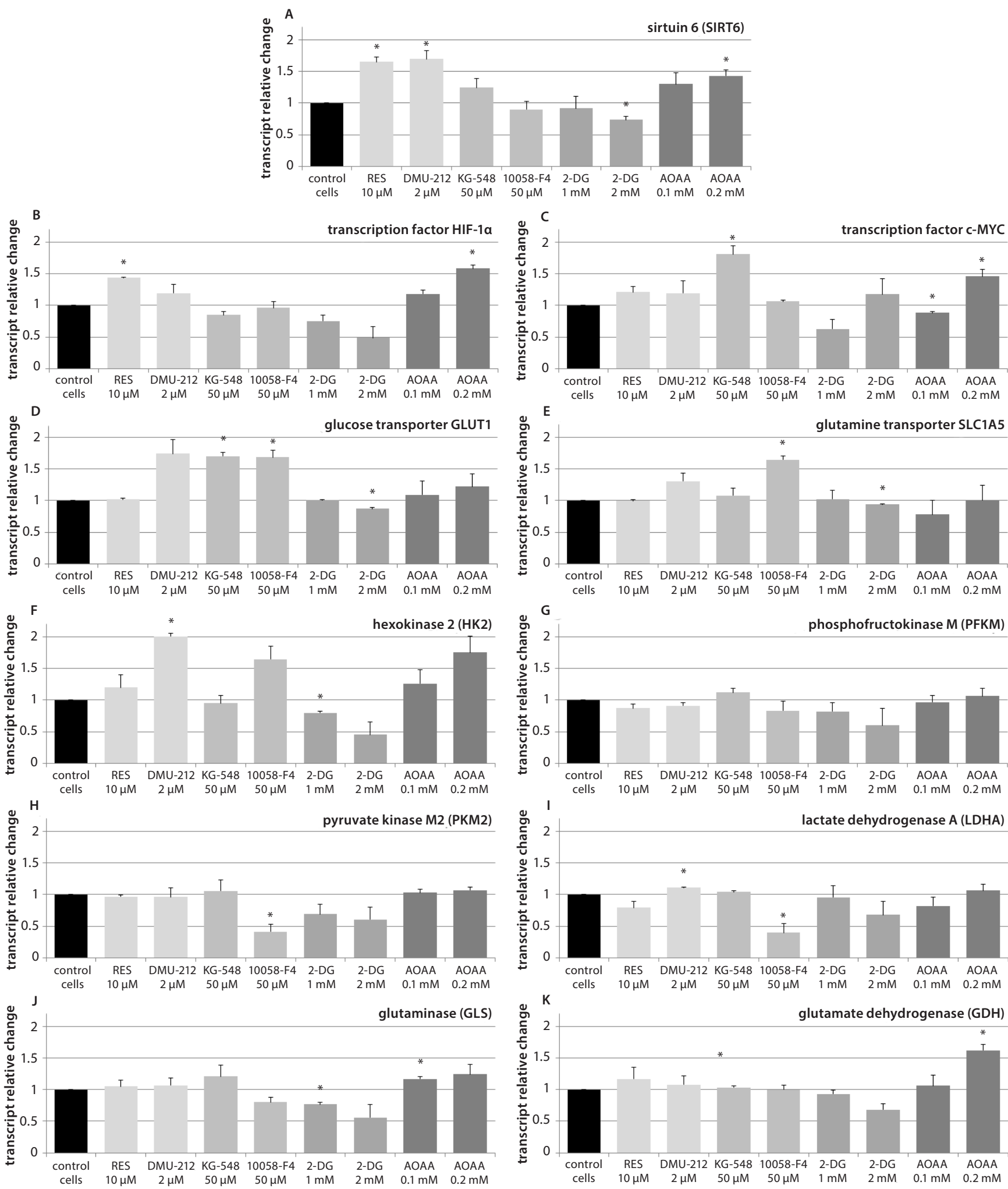

Fig. 4. The effect of resveratrol (RES), DMU-212, and small molecule inhibitors KG-548, 10058-F4, 2-deoxyglucose (2-DG), and aminooxyacetic acid (AOAA) on the expression of SIRT6 (A), HIF-1a (B), C-MYC (C), glucose (D), and glutamine (E) transporters, on key glycolysis enzymes HK2 (F), PFKM (G), PKM2 (H), and LDHA (I), as well as on key glutaminolysis enzymes GLS (J) and GDH (K) in FaDu cells

Mean values +/- SEM from 2 independent experiments are shown. The level of vehicle-treated cells was considered to be 1. An asterisk above a bar denotes statistically significant differences from the control group, $\mathrm{p}<0.05$. 
the inhibition of HIF-1 by KG-548, although resulting in a reduced production of lactate, did not affect the expression of glycolytic enzymes. Thus, these results suggest that the upregulation of proto-oncogene c-MYC plays a major role in the regulation of glycolysis in FaDu cells. To a certain extent, our results also confirm the observation that glucose, not glutamine, is the dominant energy source required for the proliferation and survival of $\mathrm{HN}$ SCC cells. ${ }^{22}$ On the other hand, the induction of SLC1A5 by $10058-\mathrm{F} 4$ is consistent with the results of other authors who showed an elevated expression of this transporter as a response to $\mathrm{C}-\mathrm{MYC}$ inhibition in human P-493 B lymphoma and PC3 prostate cancer cells. ${ }^{12,23}$ However, the mechanism which leads to the decrease in ammonia production is elusive since it cannot be explained by changes in the gene expression level alone. It may be hypothesized that ammonia secretion is reduced passively through the blocking of lactate production, which may favor mitochondrial consumption of pyruvate and thus reduce the use of glutamine for anaplerosis.

SIRT6 has recently emerged as a novel tumor suppressor which regulates aerobic glycolysis in cancer cells. Moreover, it has been proposed that SIRT6 functions as a corepressor of HIF-1a and c-MYC by deacetylating H3K9 and H3K56 at HIF-1a and c-MYC target gene promoters, respectively. ${ }^{13,24}$

Since SIRT6 is downregulated in many tumors, including HNSCCs, SIRT6 upregulation may be expected to reverse the tumorigenic metabolic profile. To test this hypothesis, we used 2 stilbene derivatives, resveratrol and its methoxy-analogue, DMU-212, as possible activators since resveratrol was previously described as an activator of SIRT1. ${ }^{25}$

Both resveratrol and DMU-212 induced the expression of SIRT6, but only slightly affected the expression of genes whose products are involved in glucose and glutamine metabolism. These results suggest that SIRT6 does not play a critical role in the regulation of the transcriptional activity of HIF-1 and c-MYC in FaDu cells. However, more detailed studies are required to confirm this observation.

Among the other modulators used in this study, 2-deoxyglucose effectively inhibited glycolysis as expected, leading to a decreased viability of FaDu cells. This observation indicates that targeting glycolysis may be a valid anti-proliferative strategy in HNSCCs, as has been recently suggested. ${ }^{26}$ The anti-proliferative effects of 2-deoxyglucose are related to blocking the consumption of glucose in glycolysis and the depletion of the cellular ATP level due to the accumulation of 2-deoxyglucose-6-phosphate (2-DG-6-P), which can neither be transformed into fructose derivative nor utilized in the pentose phosphate pathway. ${ }^{27,28}$ Accumulated 2-DG-6-P may reach levels 20-fold higher than G-6-P concentrations. 2-deoxyglucose also affects the protein $\mathrm{N}$-glycosylation, induces the accumulation of misfolded proteins, and disrupts proper protein translocation to the cell membrane. ${ }^{29,30}$
AOAA, a glutaminolysis inhibitor, also reduced $\mathrm{FaDu}$ cell viability; however, this effect cannot be attributed to the modulation of either glutaminolysis or glycolysis. AOAA's lack of effect on ammonia production may be related to the induction of glutamate metabolism by glutamate dehydrogenase, which may be associated with the induction of c-MYC expression. ${ }^{31}$ Thus, our results support the hypothesis that AOAA mediates cytotoxic effects through a stress response pathway. ${ }^{32}$

Overall, the results of the study indicate that the c-MYC transcription factor, rather than HIF-1 $\alpha$, plays a major role in the regulation of glycolytic and glutaminolytic enzyme expression in head and neck carcinoma cells. This suggests that c-MYC inhibitors, as with 2-deoxyglucose, may show anti-proliferative activity in HNSCCs by modulating dysregulated metabolic pathways.

\section{References}

1. Warburg O. On the origin of cancer cells. Science. 1956;123:309-314.

2. Hanahan D, Weinberg RA. Hallmarks of cancer: The next generation. Cell. 2011;144:646-674.

3. Samudio I, Fiegl M, Andreeff M. Mitochondrial uncoupling and the Warburg effect: Molecular basis for the reprogramming of cancer cell metabolism. Cancer Res. 2009;69:2163-2166.

4. Ward PS, Thompson CB. Signaling in control of cell growth and metabolism. Cold Spring Harb Perspect Biol. 2012;4(7):a006783. doi: 10.1101/cshperspect.a006783

5. Dando I, Fiorini C, Pozza ED, et al. UCP2 inhibition triggers ROS-dependent nuclear translocation of GAPDH and autophagic cell death in pancreatic adenocarcinoma cells. Biochim Biophys Acta. 2013;1833:672-679.

6. Wang $\mathrm{HJ}$, Hsieh YJ, Cheng WC, et al. MJD5 regulates PKM2 nuclear translocation and reprograms HIF-1a-mediated glucose metabolism. Proc Natl Acad Sci USA. 2014;111:279-284.

7. Bell EL, Emerling BM, Ricoult SJ, Guarente L. SirT3 suppresses hypoxia inducible factor $1 a$ and tumor growth by inhibiting mitochondrial ROS production. Oncogene. 2011;30:2986-2996.

8. Hammoudi N, Ahmed KB, Garcia-Prieto C, Huang P. Metabolic alterations in cancer cells and therapeutic implications. Chin J Cancer. 2011;30:508-525.

9. Kim JW, Dang CV. Cancer's molecular sweet tooth and the Warburg effect. Cancer Res. 2006;66:8927-8930.

10. Sandulache VC, Myers JN. Altered metabolism in head and neck squamous cell carcinoma: An opportunity for identification of novel biomarkers and drug targets. Head Neck. 2012;34:282-290.

11. Wise DR, DeBerardinis RJ, Mancuso A, et al. Myc regulates a transcriptional program that stimulates mitochondrial glutaminolysis and leads to glutamine addiction. Proc Natl Acad Sci USA. 2008;105:18782-18787.

12. Gao P, Tchernyshyov I, Chang TC, et al. c-Myc suppression of miR$23 \mathrm{a} / \mathrm{b}$ enhances mitochondrial glutaminase expression and glutamine metabolism. Nature. 2009;458:762-765.

13. Kleszcz R, Paluszczak J, Baer-Dubowska W. Targeting aberrant cancer metabolism - The role of sirtuins. Pharmacol Rep. 2015;67:1068-1080.

14. Nakagawa T, Guarente L. Sirtuins at a glance. J Cell Sci. 2011;124:833-838.

15. Zhong L, Mostoslavsky R. SIRT6: A master epigenetic gatekeeper of glucose metabolism. Transcription. 2010;1:17-21.

16. Sebastián C, Zwaans BM, Silberman DM, et al. The histone deacetylase SIRT6 is a tumor suppressor that controls cancer metabolism. Cell. 2012;151:1185-1199.

17. Lyssiotis CA, Cantley LC. SIRT6 puts cancer metabolism in the driver's seat. Cell. 2012;151:1155-1156.

18. Lai CC, Lin PM, Lin SF, et al. Altered expression of SIRT gene family in head and neck squamous cell carcinoma. Tumor Biol. 2013;34:1847-1854.

19. Kim HS, Xiao C, Wang RH, et al. Hepatic-specific disruption of SIRT6 in mice results in fatty liver formation due to enhanced glycolysis and triglyceride synthesis. Cell Metab. 2010;12:224-236. 
20. Rezende TM, de Souza Freire M, Franco OL. Head and neck cancer: Proteomic advances and biomarker achievements. Cancer. 2010;116:4914-4925.

21. Pignon JP, le Maître A, Maillard E, Bourhis J. MACH-NC Collaborative Group: Meta-analysis of chemotherapy in head and neck cancer (MACH-NC): An update on 93 randomised trials and 17,346 patients. Radiother Oncol. 2009;92:4-14.

22. Sandulache VC, Ow TJ, Pickering CR, et al. Glucose, not glutamine, is the dominant energy source required for proliferation and survival of head and neck squamous carcinoma cells. Cancer. 2011;117:2926-2938.

23. Dang CV. Rethinking the Warburg effect with Myc micromanaging glutamine metabolism. Cancer Res. 2010;70:859-862.

24. Cai J, Zuo Y, Wang T, Cao Y, Cai R, Chen FL. A crucial role of SUMOylation in modulating Sirt6 deacetylation of $\mathrm{H} 3$ at lysine 56 and its tumor suppressive activity. Oncogene. 2016;35:4949-4956.

25. Price NL, Gomes AP, Ling AJ, et al. SIRT1 is required for AMPK activation and the beneficial effects of resveratrol on mitochondrial function. Cell Metab. 2012;15:675-690.

26. Sobhakumari A, Orcutt KP, Love-Homan L, et al. 2-Deoxy-d-glucose suppresses the in vivo antitumor efficacy of erlotinib in head and neck squamous cell carcinoma cells. Oncol Res. 2016;24:55-64.

27. Pietzke M, Zasada C, Mudrich S, Kempa S. Decoding the dynamics of cellular metabolism and the action of 3-bromopyruvate and 2-deoxyglucose using pulsed stable isotope-resolved metabolomics. Cancer Metab. 2014;2:9.

28. Warmoes MO, Locasale JW. Heterogeneity of glycolysis in cancers and therapeutic opportunities. Biochem Pharmacol. 2014;92:12-21.

29. Pelicano H, Martin DS, Xu RH, Huang P. Glycolysis inhibition for anticancer treatment. Oncogene. 2006;25:4633-4646.

30. Kurtoglu M, Gao N, Shang J, et al. Under normoxia, 2-deoxy-D-glucose elicits cell death in select tumor types not by inhibition of glycolysis but by interfering with $\mathrm{N}$-linked glycosylation. Mol Cancer Ther. 2007;6:3049-3058.

31. Coller HA. Is cancer a metabolic disease? Am J Pathol. 2014;184:4-17.

32. Korangath $\mathrm{P}$, Teo WW, Sadik H, et al. Targeting glutamine metabolism in breast cancer with aminooxyacetate. Clin Cancer Res. 2015;21:3263-3273. 